\title{
COMUNICAÇÃO
}

\section{COMPARISON OF VIGILANCE METHODS IN THREE HOUSES WITH DIFFERENT TRIATOMA INFESTANS DENSITIES}

\author{
Marco Tulio García-Zapata, Domingos das Virgens and Philip Davis Marsden.
}

\begin{abstract}
Various vigilance methods were compared for 103 days in houses with different Triatoma infestans densities. The methods used were manual capture, capture by the inmates with plastic bag notification, the Gomez Nunez box, the Cohen trap, and paper sheets to detect faecal contamination and painted wall strips with a similar objective. It was concluded that plastic'bag notification and paper sheets are two methods of longitudinal vigilance which could be useful in national control programmes.
\end{abstract}

Key words: Triatoma infestans. Control programmes. Vigilance methods.

Various vigilance methods exist which can be installed in bug infested houses, after the attack phase with residual insecticides, during a Chagas' control programme.

We have already reported the use of a vigilance unit for individual householders ${ }^{3}$ which incorporates two effective vigilance methods namely the Gomez Nuñez box and plastic bag notification by the householder. Unfortunately the Gomez Nuñez trap is too complicated and expensive for use in routine control programmes. Since faeces are the most common sign of bug colonisation in the Gomez Nuñez box we have evaluated the use of simple paper sheets tacked to the walls with favourable results ${ }^{1}$. In this paper we evaluate these methods with two further options, namely a strip of wall surface to detect recent faecal contamination and the use of the recently developed Cohen trap.

\section{MATERIAL AND METHODS}

Since normal bug densities no longer exist in our study area of Mambai, Goiás, we studied three houses with different bug densities in Brejão farm in the municipality of Posse. This farm is divided from the east limit of the municipality of Mambai by the river Corrente. After a timed manual capture for $T$. infestans and with the house owners permission the

Núcleo de Medicina Tropical e Nutrição, Universidade de Brasilia, 70910 Brasília, DF.

Recebido para publicação em 9/11/84. following vigilance methods were installed in two houses with moderate ( 12 bugs per man hour) and high density infections ( 24 bugs per man hour).

a) 1 vigilance unit. One and half metres above the principal bed consisting of a Gomez Nuñez trap with a plastic bag.

b) 4 Cohen traps. In corners, and above beds in rooms containing bugs.

c) 4 paper sheets $(32 \times 22 \mathrm{~cm})$ were tacked to walls in rooms containing bugs.

d) Two wall surfaces $(32 \times 22 \mathrm{~cm})$ were painted with white paint in rooms containing bugs.

The paper sheets (c) were of different colours (white, yellow, green, brown, red). The Cohen trap is basically a modified Gomez Nuñez box made longer and narrow to fit into a wall angle. It is not filled with paper like the Gomez Nuñez box but instead the inside is coated with a formula of $2.5 \%$ permethrin and $2 \%$ sodium silicate.

In the house with the lowest bug density (2 bugs/man hour) only the paper sheets and painted wall strips were installed in the same numbers as above.

The houses were visited at 15 and 34 days after the vigilance methods were in place to gauge acceptance by the householders and to collect and replace the plastic bags. At 72 days the traps and paper sheets were examined for evidences of bug presence. After a further examination at 103 days the experiment was terminated. 
Comunicação. Garcia-Zapata MT, Virgens D, Marsden PD. Comparison of vigilance methods in three houses with different Triatoma infestans densities. Revista da Sociedade Brasileira de Medicina Tropical 18: 183-186, Jul-Set, 1985

Table 1 - Plastic bag collection of Triatoma infestans by householders retrieved at different times during the study.

\begin{tabular}{ccccc}
\hline $\begin{array}{c}\text { No of days collection } \\
\text { made after start of } \\
\text { the study }\end{array}$ & \multicolumn{2}{c}{ High density house } & \multicolumn{2}{c}{ Moderate density house } \\
\hline 15 & Adults & Larvae & Adults & Larvae \\
34 & 14 & 7 & 2 & 2 \\
72 & 15 & 9 & 8 & 3 \\
103 & 34 & 16 & 4 & 4 \\
\hline Totals & 35 & 14 & 3 & 13 \\
\hline
\end{tabular}

\section{RESULTS}

Although the situation was artificial in the sense of having so many vigilance methods in three houses the householders did not complain. The plastic bag collections revealed good collaboration and are set out in Table 1. The inmates of the high density house caught a total of 144 bugs in 103 days. The timed manual captures performed by us at the end of study did show differences from the initial apparent density figures suggesting that manual capture by the inmates could be responsible, for this difference. In the low bug density house only two $T$. infestans were captured in one man hour before the study began and none at the end. Yet the inmates provided eight adults in matchboxes at 15 days and three adults and one nymph at 34 days although they caught no further bugs.

Table 2 - Comparison of vigilance methods in three houses with different Triatoma infestans densities.

\begin{tabular}{lcc}
\hline $\begin{array}{l}\text { Bug capture/man hour at } \\
\text { begining of experiment }\end{array}$ & $\begin{array}{c}\text { High density } \\
28 / \text { man hour }\end{array}$ & $\begin{array}{c}\text { Medium density } \\
12 / \text { man hour }\end{array}$ \\
\hline Results at 72 days & & + \\
Gomez Nuñez box & + & $\mathrm{Neg}$ \\
Cohen traps (4) & + & + \\
Paper sheet (4) & $\mathrm{Neg}$ & $\mathrm{Neg}$ \\
Painted wall strip (2) & + & + \\
Plastic bag & & + \\
Results at 103 days & + & + \\
Gomez Nuñez box & + & + \\
Cohen traps (4) & + & + \\
Paper sheets (4) & + & + \\
Painted wall strip & + & $5 /$ man hour \\
Plastic bag & & \\
Bug capture/man hour at end & $20 /$ man hour & \\
of experiment &
\end{tabular}

The results of the various vigilance methods in the two houses are set out in Table 2. There was little to choose between the Gomez Nuñez box, paper sheet and the Cohen trap: all registered bug presence. The colour of the paper sheet did not influence evidence of bug presence. The painted wall strip finally registered faeces in the most infested house. The results clearly show that in the highly infested house eventually any method is positive. In the low density infested house on the other hand none of the papers or painted surfaces were positive. 
Comunicação. Garcia-Zapata MT, Virgens D, Marsden PD. Comparison of vigilance methods in three houses with different Triatoma infestans densities. Revista da Sociedade Brasileira de Medicina Tropical 18: 183-186, Jul-Set, 1985

Table 3 - Results of Cohen trap captures compared with plastic bag captures.

\begin{tabular}{rccccc} 
& \multicolumn{2}{c}{ High density } & \multicolumn{2}{c}{ Medium density } \\
& Plastic bag & Cohen traps & Plastic bag & Cohen traps \\
\cline { 2 - 6 } 72 days & 50 & $7 *$ & 8 & 0 \\
103 days & 49 & 8 & 7 & 1 \\
\hline
\end{tabular}

(*) Three of these bugs were still alive in the trap.

The results of examination of the Cohen traps as compared with householder manual capture are compared in Table 3. The latter proved more productive. Of the total of 16 bugs found in the Cohen trap four were still alive at the time of examination. In one trap there were eggs but no adult indicating the female had left the trap alive.

\section{DISCUSSION}

The design of these studies is open to certain criticisms. For example the wall area covered by some of the vigilance methods was much greater than others in the order: Gomez Nuñez $<$ Painted surface $<$ Paper sheets $<$ Cohen traps. The total area covered by four Cohen traps was 0.32 square metres and by the Gomez Nuñez box only 0.08 square metres. Also we know from our demolition studies in Mambai ${ }^{2}$ that the distribution of $T$. infestans in the wall fabric is not uniform but is governed by the availability of blood at the wall section interface. For this reason we always recommended installing the vigilance unit above the marital bed.

A better study design would have been to compare vigilance methods at the same wall site over a standard time period. However this was not possible since the study houses were programmed for spraying with residual insecticide at the end of this study.

Among the vigilance methods the value of longitudinal collections of bugs made by the householder is demonstrated. The houses were chosen because of bug presence and the householder contacted shortly before mounting the vigilance methods. Yet the response to a demand for bug collection by the inmates is impressive.

The relative failure of the painted wall surface compared to the paper sheets may be associated with the light colour of a white painted surface which would be less attractive to bugs ${ }^{5}$. The Cohen trap was designed to reduce bug populations and not as a vigilance method. The results from this small study suggest it does not function well in either respect with T. infestans. Cohen (personal communication) found that up to $26.5 \%$ of the total population of Rhodnius prolixus present in experimental chicken houses in Venezuela could be found in the trap. We have no information on the behavior of these traps in a laboratory setting but permethrin has a "flushing out" effect on bugs and could affect trap entry ${ }^{4}$. In our limited observations bugs survived in the trap and on at least one occasion left it. We see no advantage at the moment in replacing the Gomez Nunez trap with this option, although larger studies are planned.

A major objective of our studies is to establish simple more sensitive vigilance methods for detecting bug presence after the attack phase in national control programmes. Manual capture is expensive in time and money (field allowance, gasoline) for a 15 minute attempt at capture in houses where bug populations will often be below detectable levels. We believe that at least in $T$. infestans areas plastic bag collection by the householder and a yearly examination of paper sheets tacked over the principal beds could be more sensitive and cheaper.

\section{RESUMO}

Vários métodos de vigilância foram comparados em casas com diferentes densidades de Triatoma infestans por 103 dias. Os métodos utilizados foram a captura manual, captura feita pelos moradores com notificação dentro da sacola plástica, caixa de Gomez Nuñez, armadilha de Cohen, folhas de papel afixadas na parede para detectar a contaminação fecal e faixas pintadas na parede como mesmo objetivo. Foi concluido que a notificação da sacola plástica e as folhas de papel são dois métodos de vigilância longitudinal que podem ser úteis na programação nacional de controle.

Palavras-chave: Triatoma infestans. Programas de controle. Métodos de vigilância. 
Comunicafão Garcia-Zapata MT, Virgens D, Marsden PD. Comparison of vigilance methods in three houses with different Triatoma infistans densities. Revista da Sociedade Brasileira de Medicina Tropical 18: 183-186, Jul-Set, 1985

\section{ACKNOWLEDGEMENTS}

We are grateful to Antonio Carlos Silveira (Director of the Chagas' Disease Control Programme), Carlos Alberto Matos (Director of SUCAM, Goiás) and Alfredo Oliveira Filho (Rio de Janeiro) for permission to work in Brejão, and to Dean H.L. Cohen, School of Architecture and Enviromental Design, Buffalo, New York for furnishing his traps evaluated in this study. These studies were supported by the Conselho Nacional de Desenvolvimento Cientifico e Tecnológico do Brasil (CNPq grant no 403032/82) and the UNDP/World Bank/WHO Special Programme for Research and Training in Tropical Diseases.

\section{REFERENCES}

1. Garcia Zapata MT, Schofield CJ, Marsden PD. A simple method for detecting the presence of live triatomine bugs in houses sprayed with residual in- secticide. The Transactions of the Royal Society of Tropical Medicine and Hygiene 79: 558-559, 1985.

2. Marsden PD, Alvarenga NJ, Cuba CC, Shelley AJ, Costa CH, Boreham PFL. Studies of the domestic ecology of Triatoma infestans by means of house demolition. Revista do Instituto de Medicina Tropical de São Paulo 21: 13-25, 1979.

3. Marsden PD, Penna R. A vigilance unit for households subject to tritomine control. The Transactions of the Royal Society of Tropical Medicine and Hygiene 76: 790-792, 1982.

4. Pinchin R, Oliveira Filho AM, Pereira ACB. The flushing-out activity of pyrethrum and synthetic pyrethroids on Panstrongylus megistus, a vector of Chagas's disease. The Transactions of the Royal Society of Tropical Medicine and Hygiene 74: 801-803, 1980.

5. Schofield CJ. The behavior of triatominae (Hemiptera, Reduviidae): a review. Bulletin of Entomological Research 69: 363-379, 1979. 